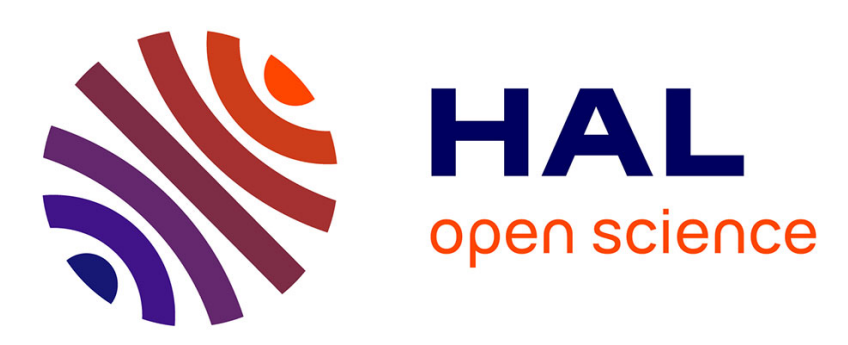

\title{
Determination of the temperature dependent thermal conductivity of isotopically modified single crystal diamond
}

\author{
Lumei Wei, P. Kuo, R. Thomas
}

\section{To cite this version:}

Lumei Wei, P. Kuo, R. Thomas. Determination of the temperature dependent thermal conductivity of isotopically modified single crystal diamond. Journal de Physique IV Proceedings, 1994, 04 (C7), pp.C7-229-C7-232. 10.1051/jp4:1994754 . jpa-00253283

\section{HAL Id: jpa-00253283 https://hal.science/jpa-00253283}

Submitted on 1 Jan 1994

HAL is a multi-disciplinary open access archive for the deposit and dissemination of scientific research documents, whether they are published or not. The documents may come from teaching and research institutions in France or abroad, or from public or private research centers.
L'archive ouverte pluridisciplinaire HAL, est destinée au dépôt et à la diffusion de documents scientifiques de niveau recherche, publiés ou non, émanant des établissements d'enseignement et de recherche français ou étrangers, des laboratoires publics ou privés. 


\title{
Determination of the temperature dependent thermal conductivity of isotopically modified single crystal diamond
}

\author{
L. Wei, P.K. Kuo and R.L. Thomas \\ Department of Physics and Astronomy and Institute for Manufacturing Research, Wayne State University, \\ Detroit, Michigan, 48201, U.S.A.
}

\begin{abstract}
The experimental details of the in-solid mirage technique for the determination of thermal conductivities of diamond crystals of different isotopic compositions at low temperatures is described. The significance of the results is discussed.
\end{abstract}

\section{INTRODUCTION}

In the study of crystal lattice dynamics in solid state physics, diamond offers a very unique proving ground. It has the smallest unit cell volume and the strongest bond strength among all naturally occurring crystals. These virtues translate into the facts that diamond is the hardest known material and has the highest thermal conductivity at room temperature. In the quest for newer and better materials, it is imperative that the theoretical understanding is verified on familiar materials. In particular, the theory of the dependence of thermal conductivity of dielectric crystals on the isotopic concentration, which plays a central role in the understanding of phonon scattering by lattice imperfections, has been in a fairly advanced stage since the fifties. However, the experimental verification has been limited to crystals like $\mathrm{LiF}$ and $\mathrm{Ge}$, where the isotopic effect is not more than a few percent. Some subtle aspects of the theory had never been seriously challenged. Diamond would have been a good testing material, but, until recently, it had been available only in the natural isotopic abundance of carbon, namely, $98.9 \% \mathrm{C}^{12}, 1.1 \% \mathrm{C}^{13}$ and negligible amounts of $\mathrm{C}^{14}$. The wide spread usage of tracer chemistry in biological science brought the commercial availability of isotopically modified carbon in the form of methane, thus making it possible to grow synthetic diamonds of various isotopic compositions. General Electric Corporate R\&D laboratory did grow such crystals. The initial measurements of thermal conductivity at room temperature on these crystals indicated a very strong dependence on isotopic composition[1-3]. However, attempts to correlate the experimental findings with theory did not succeed[2-4], mostly due to the lack of data at lower temperature region and a confusion over what aspects of the theory should be included. A recent report[5] presented not only results of thermal conductivity measurements of diamond crystals of different isotopic compositions at low temperature, but also presented an analysis that showed that all existing data on thermal conductivity of diamond are in good agreement with existing theory. This paper reports on the experimental technique used in Ref. [5].

\section{EXPERIMENTAL METHODS}

From the knowledge of the Debye temperature of diamond it is easy to estimate the gross behavior of its thermal conductivity. The conductivity is expected to increase steeply from room temperature, 
reaching a peak roughly 10 times that at room temperature at roughly 80-100 K. Our measurement technique used in the earlier experiment[1] was that of thermal wave generation, using the mirage effect as the temperature probe in the atmosphere immediately over the specimen. By knowing the frequency and measuring the wave length of the thermal wave, the thermal diffusivity can be directly determined without calibration procedures which usually accompany conventional thermal measurements. However, this method is deemed inadequate in the low temperature region, where the thermal diffusivity is expected to increase 3 orders of magnitude, due to the rapidly falling thermal capacity and rising thermal conductivity. The wave length of the thermal wave at convenient modulation frequencies could easily exceed the physical dimension of the specimens. If the frequency is increased to produce shorter thermal waves, then the corresponding attenuation of the thermal waves in the atmosphere would become intolerable. Another complication is the Kapitza resistance at the interface between the metal coating and diamond. The function of the metal coating is to convert optical energy into thermal energy. With the in-air mirage detection, the temperature measurement is made on the side of coating opposite to diamond. Thus, the Kapitza resistance is expected to be a contributing factor at temperatures much lower than room temperature where it is not negligible. Owing to these considerations, the in-solid mirage effect[6] was selected as the temperature probe. Since the temperature gradient inside the crystal is measured directly, the thermal diffusivity can be deduced without the thermal wave having to cross any interface or boundary. In deducing physical parameter from experimental data, one needs to solve the thermal diffusion equation in one medium only. The complication of the boundary or interface is thus avoided.

The experiment is carried out in a liquid helium dewar having three optical windows for the passage of the probe and heating laser beams. Two specimens were used in this experiment. They both have dimensions $1 \times 2 \times 4 \mathrm{~mm}$. One has natural isotopic composition, while the other one is isotopically enriched to contain $99.9 \% \mathrm{C}^{12}$ and $0.1 \% \mathrm{C}^{13}$. The natural diamond crystal was purchased from Double Dee Harris Co. and is of type IIA. The isotopically enriched specimen was grown at the General Electric Corporate R\&D laboratory and is also of type IIA. Both specimens had one of their small ends metallized to serve as the heat source when illuminated by a focused Art laser beam. The opposite end is cemented to a copper block sample holder which is placed in a liquid helium dewar. Near the cemented end a fine wire $(50 \mu \mathrm{m})$ thermal couple is wrapped around the specimen as the ambient temperature monitor. On the sample holder there is a Si diode temperature sensor whose output is used as the feedback signal in the temperature control system. The difference between the readings of the two temperature sensors allows us to monitor the average thermal flux through the specimen. The resistance heater wire of the temperature control system is also attached to the copper sample holder near the temperature sensor diode. Even when the experiment was carried out at temperatures above $77 \mathrm{~K}$, it was still necessary to use liquid helium because the heat load was quite high, exceeding one watt much of the time.

The simplest way to measure the diffusivity is to measure the exponential decay of the amplitude and the linear phase variation along the direction of propagation of a planar thermal wave. To achieve this, preferably one would either place the source at a large distance away or would use a uniform source. With the restriction of our specimen geometry, neither is feasible for this experiment. The over-all length of the specimens is very small to begin with. To have a uniform source would imply the use of a Gaussian beam larger than the lateral size of the specimens. This would entail the difficulty of shielding and dissipating the unused optical power that spills over the end face of the specimen. An additional complication is that the ends are not perfectly rectangular shaped, due to damage during cutting and polishing. It was decided to use a Gaussian beam of about $100 \mu \mathrm{m}$ in radius, and to carry out a computation of the wave form of the thermal wave in a narrow channel with reflecting boundary conditions. In order to maximize the mirage signal, the probe beam is made to traverse the direction of the $2 \mathrm{~mm}$ dimension. This leaves the narrow $1 \mathrm{~mm}$ dimension as the channel width in which we solve the diffusion equation for a damped Gaussian thermal wave. We choose the coordinate axes such that the $z$-axis is along the long dimension of the specimen and the $x$-axis along the shortest. The direction of propagation of the probe beam is then the y-axis. We made several lateral scans (in the $x$-direction) at different values of $\mathrm{z}$ (distance from the source of the thermal wave). The amplitude and phase of the gradient along $\mathrm{z}$ are compared in Fig. 1 to show that the waves behaved as expected. Note that in this region the waves are neither planar nor Gaussian. 
To establish correspondence with the in-air mirage method, we used the in-solid mirage method to measure the diffusivity of both specimens at room temperature. Figure 2 shows the amplitude and phase of the nomal deflection as functions of the distance of thermal wave propagation (z-coordinate), as compared to the theoretically predicted behavior based on diffusivity values measured with the in-air method, namely, $12.8 \mathrm{~cm}^{2} / \mathrm{s}$ and $17.7 \mathrm{~cm}^{2} / \mathrm{s}$ for the natural and enriched diamond specimens, respectively. In Fig. 3 we show some typical amplitude and phase data at two temperatures.

\section{RESULTS AND DISCUSSIONS}

The main results of this analysis have been reported earlier[5]. The major conclusion is that our room temperature and low temperature data fit very well with the theory proposed by Callaway[7]. We were able to determine the empirical parameters (the scattering rates) in the theory by fitting that theory our data. With these parameters determined, the Callaway theory then gives a complete formula for the thermal conductivity of diamond of any isotopic composition, of any size, and at any temperature. All the previously existing data on type IIA diamond, that include the high temperature data by Vandersande et al[8] and the low temperature data by Berman et al[9], also agree very well with this formula. This formula further predicts that, at room temperature, the isotopic enhancement saturates at about $50 \%$ near the purity achieved by the GE specimen $(99.9 \%)$. No further enhancement at room temperature should be expected by achieving higher isotopic purity. However, at a low temperature of $80 \mathrm{~K}$ (still above the liquid nitrogen boiling point), the saturation is reached at $99.999 \%$ isotopic purity with a thermal conductivity of well over $2 \times 10^{5} \mathrm{~W} / \mathrm{MK}$.

A salient feature of Callaway's theory is the inclusion of the normal (N-) processes of phonon scattering. Even though the N-processes do not contribute to thermal resistance directly, because they preserve lattice momentum, they play a crucial role in determining the phonon mean-free-path when the rate of umklapp (U-) processes is low. This is the case for type IIA diamond for temperatures below $400 \mathrm{~K}$.

Using conventional techniques, this experiment would have been difficult, if not impossible, with the restriction of specimen geometry. With the in-solid mirage technique, the measured thermal conductivity values have been determined unambiguously without the uncertainty of effects occurring at interfaces and boundaries. The results enabled us to determine the phonon-phonon scattering rates for $\mathrm{N}$ - and U-processes, which have been the missing links of a consistent theoretical picture.

\section{REFERENCES}

[1] T.R. Anthony, W.F. Banholzer, J.F. Fleischer, Lanhua Wei, P.K. Kuo, R.L. Thomas, and R.W. Pryor, Phys. Rev. B42 (1990) 1104.

[2] D.G. Onn, A. Witek, Y.Z. Qiu, T.R. Anthony, and W.F. Banholzer, Phys. Rev. Lett. 68 (1992) 2806.

[3] J.R. Olson, R.O. Pohl, J.W. Vandersande, A. Zoltan, T.R. Anthony, and W.F. Banholzer, Phys. Rev. B (to be published).

[4] J.W. Bray and T.R. Anthony, Z. Phys. B84, 51 (1991).

[5] Lanhua Wei, P.K. Kuo, R.L. Thomas, T.R. Anthony, and W.F. Banholzer, Phys. Rev. Lett. 24 (1993) 3764.

[6] W.B. Jackson, N.M. Amer, A.C. Boccara, and D. Fournier, Appl. Opt. 20 (1981) 1333.

[7] J. Callaway, Phys. Rev. 113 (1959) 1046.

[8] J.W. Vandersande, A. Zoltan, J.R. Olson, R.O. Pohl, T.R. Anthony, and W.F. Banholzer, Prod. VII Intl. Conf. on Scattering of Phonons in Condensed Matter, Aug. 3-7, 1992, Cornell University.

[9] R. Berman and J.C.F. Brock, Proc. Roy. Soc. A, 289 (1965) 46. 

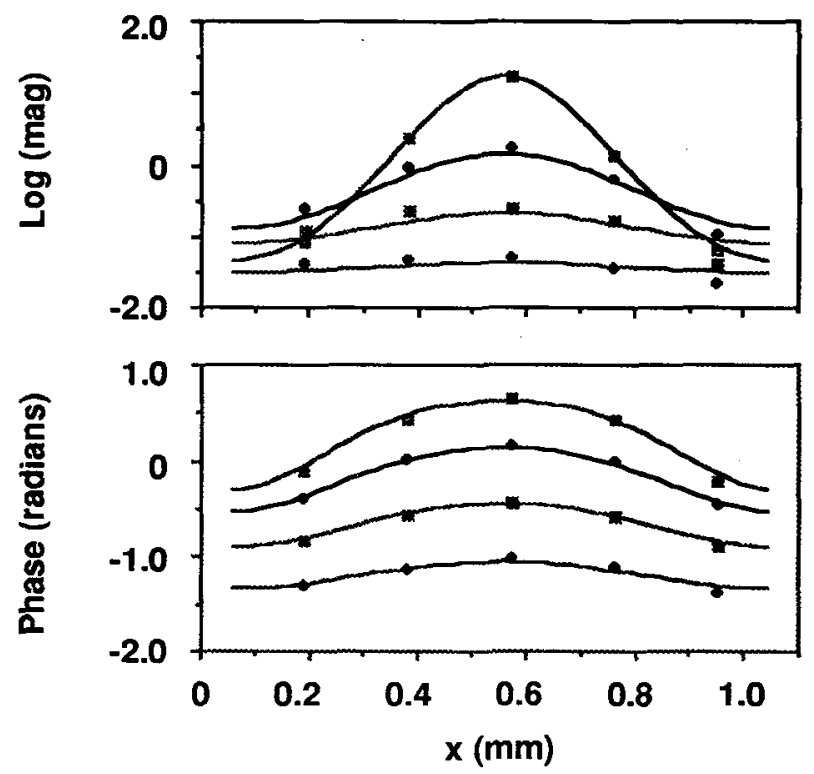

Fig. 1 Comparison between theory (lines) and experiment (symbols) for the lateral profile of the in-solid mirage signal at $3.6 \mathrm{kHz}$ and the radius of the heating beam is $90 \mu \mathrm{m}$. The $z$ values for the four curves from top to bottom are 126,317 , 507 , and 698 , respectively.

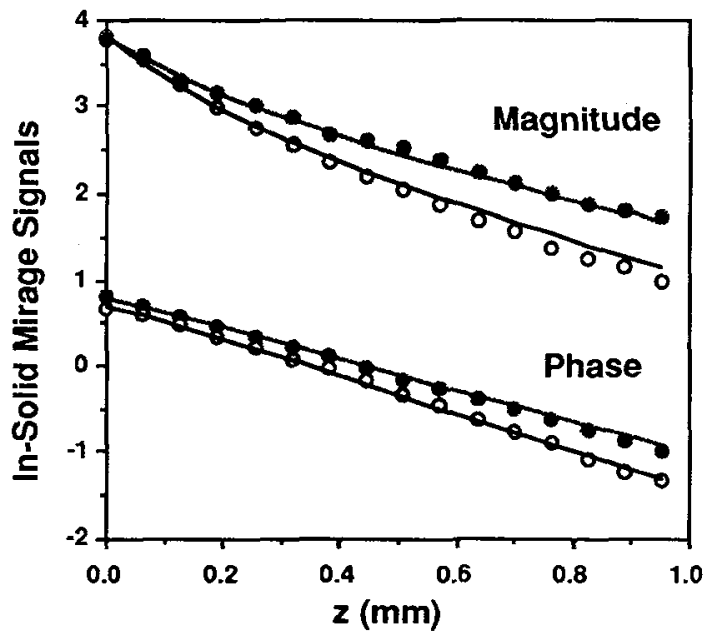

Fig. 2 Comparison of the in-solid mirage signal (magnitude and phase of the $z$-component of the temperature gradient) for the isotopically enriched diamond (upper curves, diffusivity $=17.7 \mathrm{~cm}^{2} / \mathrm{s}$ ) and natural abundance diamond (lower curves, diffusivity $=12.8 \mathrm{~cm}^{2} / \mathrm{s}$ ) at $298 \mathrm{~K}$.

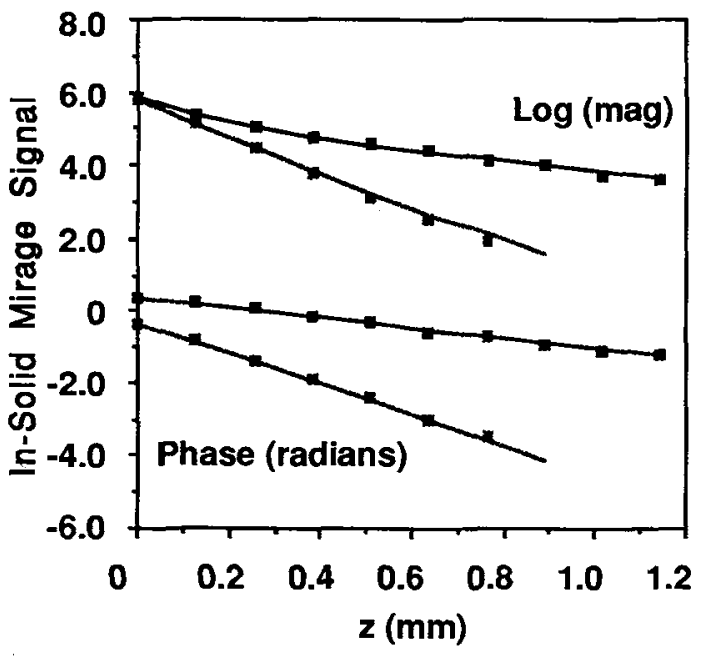

Fig. 3 Comparison of the in-solid mirage signal (magnitude and phase of the $\mathrm{z}$-component of the temperature gradient) for the natural abundance diamond at $175 \mathrm{~K}$ (upper curves, diffusivity $=121$ $\mathrm{cm}^{2} / \mathrm{s}$ ) and $298 \mathrm{~K}$ (lower curves, diffusivity $=12.6$ $\mathrm{cm}^{2} / \mathrm{s}$ ). Modulation frequency is $6.4 \mathrm{kHz}$. 